Article

\title{
The Effects of High Fat Diet-Induced Stress on Olfactory Sensitivity, Behaviors, and Transcriptional Profiling in Drosophila melanogaster
}

\author{
Jewon Jung, Dong-In Kim, Gi-Youn Han $₫$ and Hyung Wook Kwon * \\ Department of Life Sciences and Convergence Research Center for Insect Vectors, Incheon National University, \\ 119 Academy-ro, Yeonsu-gu, Incheon 22012, Korea; jjewony@gmail.com (J.J.); kdin34@naver.com (D.-I.K.); \\ bkdgy93@gmail.com (G.-Y.H.) \\ * Correspondence: hwkwon@inu.ac.kr; Tel.: +82-32-835-8090
}

Received: 17 July 2018; Accepted: 12 September 2018; Published: 20 September 2018

check for updates

\begin{abstract}
High-fat diet (HFD) often causes obesity and it has detrimental effects on the sensory system. In particular, sensory-mediated responses are crucial for maintaining energy balance, as they are involved in a metabolic regulation; however, there is still no clear explanation about the relationship between HFD-induced stress and sensory system. To gain insight on how HFD-induced stress affects olfactory sensitivity and behavioral responses, we have used a Drosophila melanogaster model for olfactory and nutrient-related signaling and accessed physiological, behavioral, and transcriptional changes. We demonstrated that lifespan and climbing ability in HFD-treated flies decreased and that olfactory sensitivity and behavioral responses to odorants were changed. Olfactory sensitivity to eight of ten odorants after 14 days on HFD treatment were reduced, while behavioral attraction was increased to benzaldehyde in flies that were treated with HFD. This behavioral and physiological modification in HFD-treated flies for 14 days was accompanied by a significant decrease in DmOrco gene expression in a peripheral olfactory organ, suggesting that is could be involved in the action of metabolic and sensory signal. Gene expression profiles of antennae showed significant differences on the olfactory receptors, odorant-binding proteins, and insulin signaling. Our results suggested that olfactory sensitivity and behavioral responses to HFD-induced stress are mediated through olfactory and nutrient-related signaling pathways.
\end{abstract}

Keywords: Drosophila melanogaster; high fat diet; olfaction; olfactory receptors; gene expression; climbing behaviors

\section{Introduction}

Most organisms maintain energy homeostasis to survive and function effectively under the influence of internal and external factors for biological processes of growth, development, metabolism, and aging [1,2]. Many environmental conditions, including nutrients, temperature, and light can influence food intake and cause consequences for health, including obesity [3,4]. Obesity causing a myriad of complex diseases is known to be related with an imbalance in the homeostasis of energy metabolism [5-7]. A previous study reported that simultaneous processing in taste and olfaction, the pivotal senses for survival, is associated with human obesity [8]. For instance, food intake controlled by external sensory signals has an effect on energy balance assessed by internal metabolic signals [9]. These fundamental responses are made through physiological changes, metabolic hormones, in particular, which regulate appetite [10].

Sensory-mediated responses are remarkably complex network [11,12]. In the natural environment, olfaction is related to finding food sources, recognizing predators, and locating mates $[13,14]$. 
In particular, feeding behaviors are closely linked and initiated with the finding of food sources in response to sensory inputs, for instance, olfactory information [12]. In mammals, such metabolic regulation in the olfactory cortex is changed by internal metabolic states where an increased number of insulin receptors (InR) on the olfactory bulb was found in starved mice [15] suggesting that the regulation of insulin signaling by food intake was attributed to olfaction.

Drosophila melanogaster, the fruit fly that is a powerful tool for studying gain- and loss-of-function by observing phenotypic changes and behavioral analysis, possesses evolutionarily conserved pathways in common with mammals [16,17]. Evidence for the link between olfactory response and insulin signaling has been shown by flies fed high-fat diet (HFD) exhibiting decreased olfactory sensitivity and odor-driven food searching behavior in comparison with flies that were fed with normal diet [18]. However, little is known about the impact of food stress on olfactory signaling and nutrient-related genes in the main peripheral olfactory organ, the antennae, of Drosophila melanogaster. It has been suggested that preferential odorant of over-weight people appeared to be a food-related rather than a non-food related [8]. Given this evidence, the alteration of metabolism and enhancement of stress resistance by the olfactory system in relation to food-derived odors might be involved in the modulation of the lifespan. Furthermore, these findings are likely to be correlated with feeding behavior and the energy balance through sensory processing [19-21].

To gain insight into how HFD-induced stress affects olfactory sensitivity and behaviors that mediate nutrient-related signaling, this study centers on the understanding of behavioral and sensory modification by food stress, such as HFD treatment in D. melanogaster, and it employs lifespan analysis, behavioral tests such as a climbing test, a choice test for food-related odorants, and electrophysiological recordings from antennae (Electroantennogram, EAG). Moreover, to pinpoint any modifications at the molecular level in the main olfactory organ, we examined the expression levels of DmOrco gene by quantitative real-time PCR (qRT-PCR) and overall transcriptional profiles of antennal tissues to identify genes including olfactory and nutrient-related signaling genes whose levels fluctuated with HFD treatment. These approaches provide a general overview of relationships between food stress and sensory modulation machineries in the peripheral olfactory organ based on Gene Ontology (GO).

\section{Results}

\subsection{Life Span and Climbing Abilities of Flies Fed with HFD}

We hypothesized that high fat diet (HFD), causing the alteration of physiological states [22,23], might reduce the life span and climbing ability of flies. To confirm the hypothesis, we fed a control diet and high-fat diet (HFD) ad libitum to male Canton-S adult flies four days after eclosion for seven days or 14 days (Figure 1A), with slight modification from the previous study [22]. The results showed that the life span of the flies fed with HFD diet started to decrease at day 7 and it was significantly reduced by approximately $12 \%$ at day 14 in comparison with those receiving the control diet (Figure 1B). Consistent with this, there were also indications that the locomotor activity of the flies declined after HFD intake. Since the diet appeared to be associated with climbing ability [24], we measured the climbing ability of flies fed with a control diet or HFD for seven or 14 days. Significant changes in climbing ability were observed for the HFD-treated groups (Figure 1C), suggesting that a HFD has a detrimental influence on fly mobility. 
A

Control diet feeding paradigm

\begin{tabular}{|c|c|c|c|}
\hline $\begin{array}{c}\text { Control } \\
\text { diet }\end{array}$ & $\begin{array}{c}\text { Control } \\
\text { diet }\end{array}$ & $\begin{array}{c}\text { Control } \\
\text { diet }\end{array}$ & $\begin{array}{c}\text { Control } \\
\text { diet }\end{array}$ \\
\hline
\end{tabular}

HFD feeding paradigm

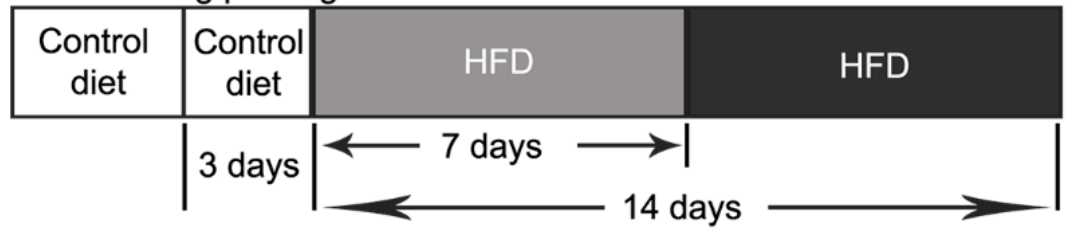

\begin{tabular}{|l|l|}
\hline Larva & Adult (Total 17 days) \\
\hline
\end{tabular}
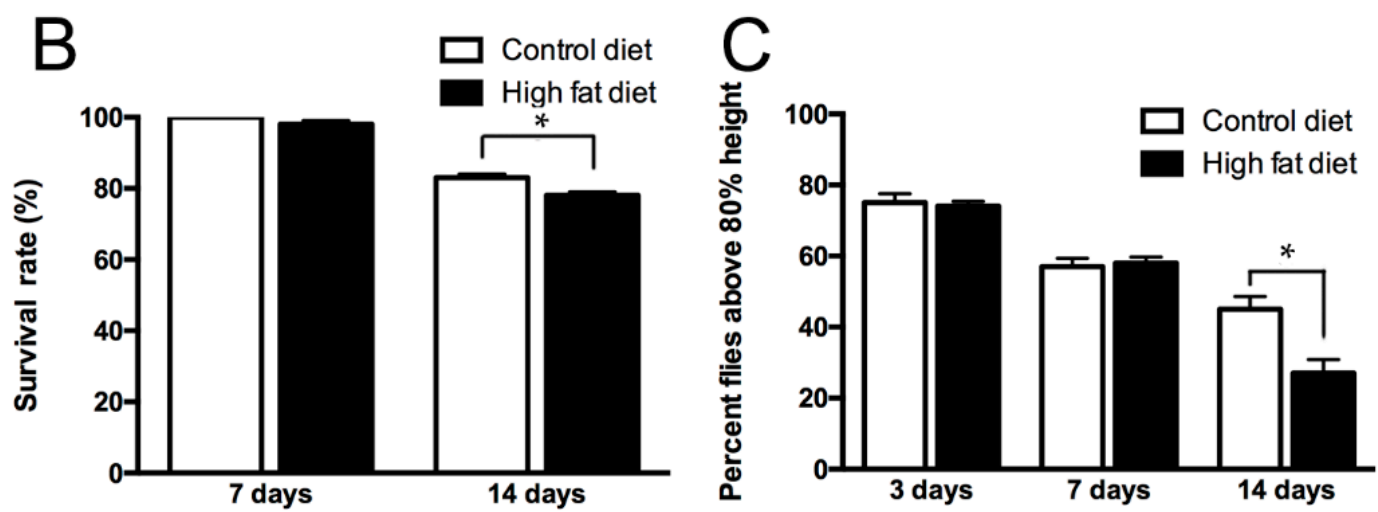

Figure 1. Physiological effects of high-fat diet (HFD) intake on wild type Canton-S flies. (A) Food treatment condition and feeding paradigm. The control diet and HFD were treated ad lib for seven and 14 days in male Canton-S flies. (B) Lifespan of wild flies fed with control diet and HFD. ( $n=5$, $\left.{ }^{*} p<0.05\right)$. Error bars represent SEM. (C) Locomotor activity of wild type Canton-S flies fed with control diet and HFD. $(n=10, * p<0.05)$. Error bars represent SEM.

\subsection{Modification of Olfactory Sensitivity by HFD Treatment}

Olfactory sensitivity is known to be affected by internal and external factors [25]. Therefore, we asked whether the HFD affects olfactory sensitivity, including attraction to food odors, such as ethyl acetate, isoamyl acetate, pentyl acetate, 2-heptanone, and benzaldehyde when compared to non-food odors such as 1-hexanol, 3-octanol, 1-octanol, 4-methyl phenol, and 4-propyl phenol. A significant difference in EAG responses was found in flies fed with HFD for seven or 14 days (Figure 2A,B). Flies treated with HFD for 14 days showed decreases in olfactory sensitivities for the food odorants and three of five non-food odorants (Figure 2B); however, the responses of acetate odorants on HFD treatment for seven days had no significant differences between flies on a HFD and standard diet (Figure 2A). It was noting that olfactory sensitivity to benzaldehyde and 1-hexanol increased substantially in flies fed with HFD for seven days (Figure 2A). 


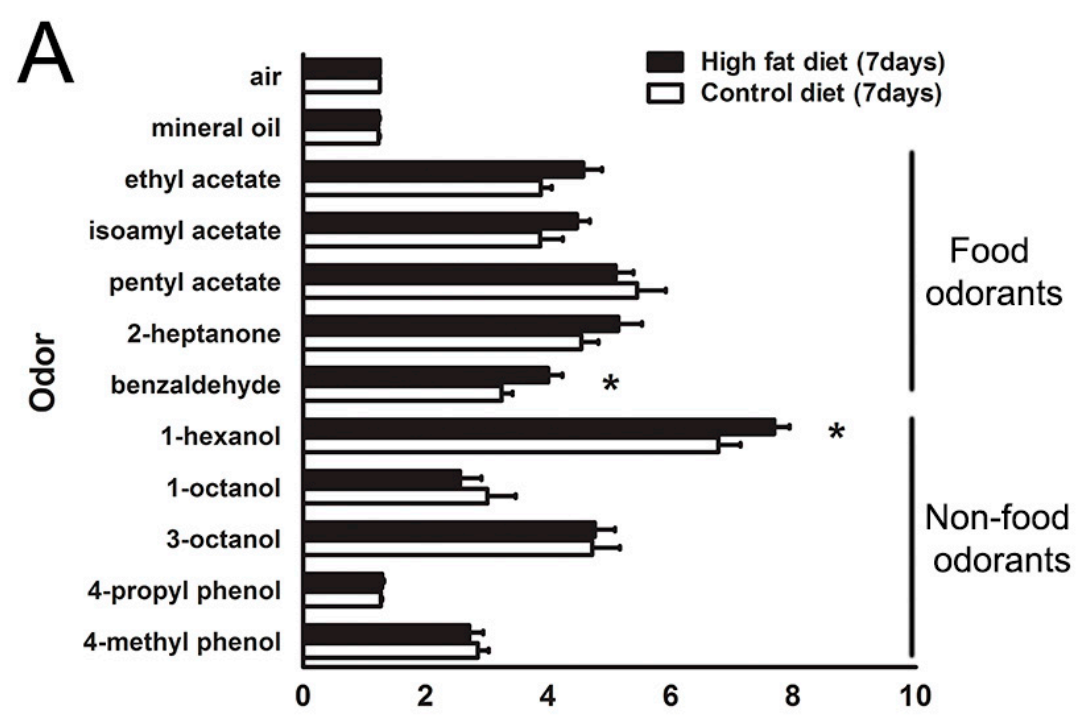

EAG amplitude (mV)

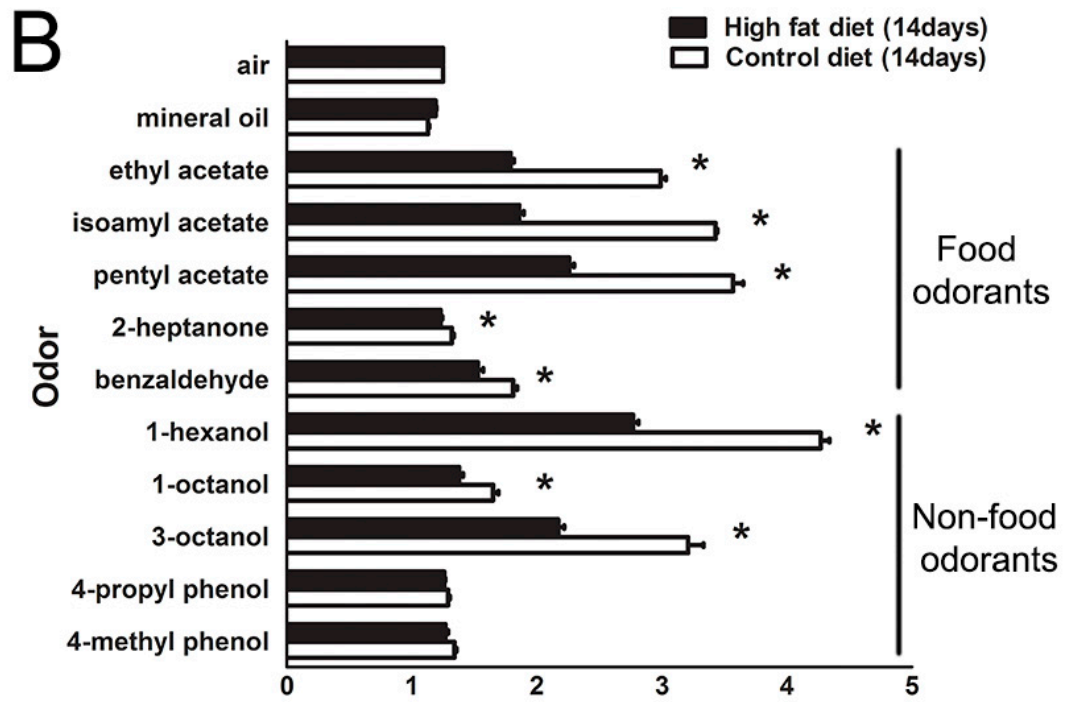

EAG amplitude (mV)

Figure 2. Olfactory responses of wild type Canton-S flies fed with control diet and HFD for seven days and 14 days. (A) Flies fed with HFD for seven days showed a reduction of olfactory responses to several odors. ( $n=15$ flies, ${ }^{*} p<0.05$ ). Error bars represent SEM. (B) Diet treatment for 14 days demonstrated that olfactory sensitivity was mostly decreased to most odorants. Flies fed with HFD for 14 days showed a decline of olfactory responses to several odors, such as ethyl acetate, isoamyl acetate, pentyl acetate, 1-hexanol, and 3-octanol. ( $n=15$ flies, ${ }^{*} p<0.05$ ). Error bars represent SEM.

\subsection{Reduction of DmOrco Gene Expression in the Fly Antenna after HFD Treatment}

We next asked whether the odorant receptor co-receptor gene of D. melanogaster (DmOrco), expressed in most olfactory receptor neurons (ORNs) [25], is affected in flies by HFD treatment. The results showed that the level of DmOrco transcripts was significantly reduced by approximately $70 \%$ in flies fed with HFD for seven days when compared with those of control flies, while it was about a $47 \%$ reduction at 14 days (Figure $3 \mathrm{~A}, \mathrm{~B}$ ). 
A

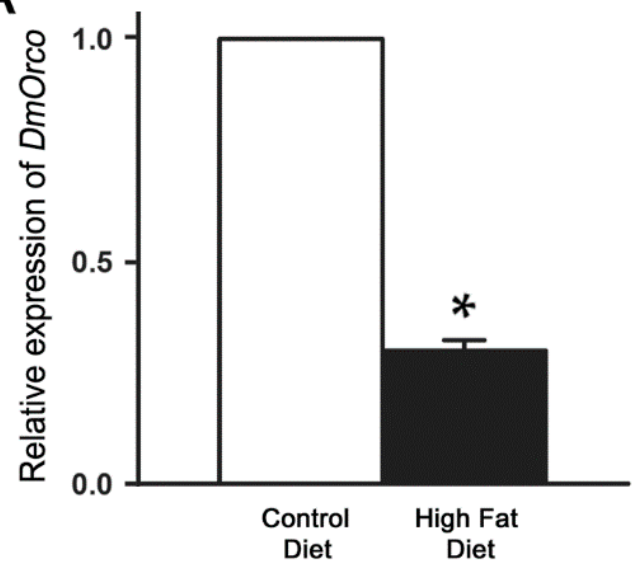

B

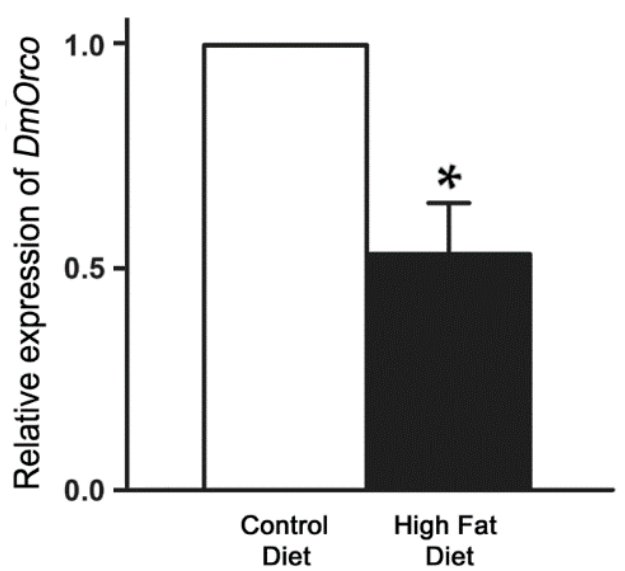

Figure 3. Relative expression of DmOrco in antenna of wild type Canton-S flies fed control diet and HFD for seven days and 14 days. (A) Relative DmOrco mRNA transcript levels after different food treatment for seven days. The level of DmOrco transcript was significantly reduced in HFD-fed flies 7 days after food treatment, showing an approximately $70 \%$ decrease compared with control flies. (B) Relative DmOrco mRNA transcript levels after different food treatment for 14 days. All qRT-PCRs were carried out in triplicate $\left.{ }^{*} p<0.05\right)$. Error bars represent SEM.

\subsection{Modification of Odorant Choice Behaviors by HFD Treatment}

HFD-fed flies showed different responses in terms of attraction and repulsion to the experimental odors as compared with those on the control diet. First, flies on a HFD at 14 days showed a significant decrease in appetitive behavior to ethyl acetate, isoamyl acetate, pentyl acetate, and 2-heptanone. On the other hand, there was an increase in repulsive behavioral responses to 3-octenol (14 days), 4-methyl phenol (7 and 14 days) in flies fed with HFD (Figure 4). In addition, the behavioral responses of HFD-fed flies to benzaldehyde and pentyl acetate showed a transition between repulsive and attractive behavior. In detail, there was an attractive behavioral response to benzaldehyde by flies fed with HFD for seven and 14 days, while the response to pentyl acetate disappeared with HFD for seven days (Figure 4). We found that the behavior of HFD-fed flies to 4-propyl phenol shift from repulsive response to appetitive response (Figure 4). In addition, while HFD-fed flies at seven days exhibit increased attractive response to 1-octanol, flies exposed to HFD at seven days show decreased attractive response to 1-octanol when compared to the response of control diet-fed files (Figure 4). Taken together, the exposure to high levels of dietary fat affects fruit fly olfactory related behavior. 

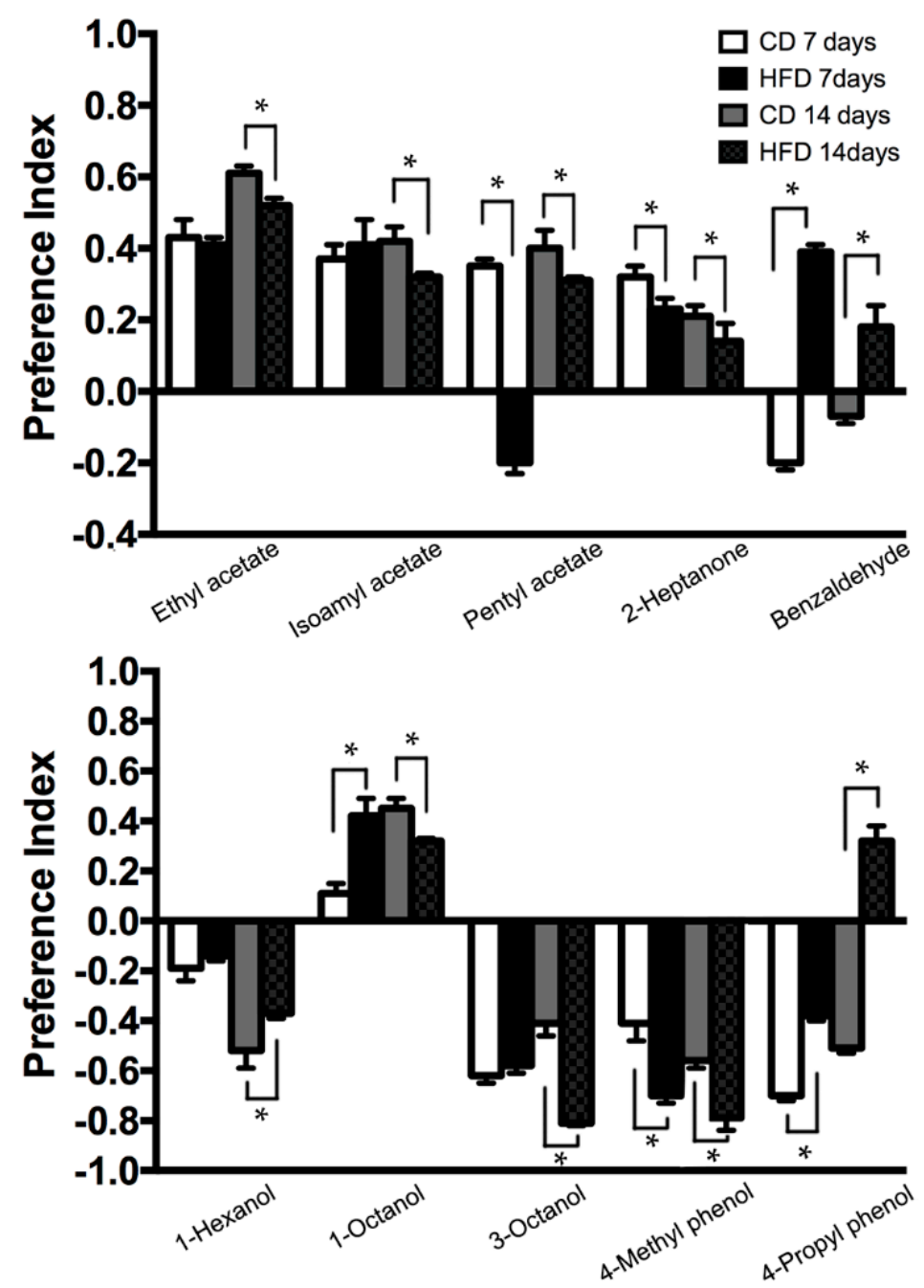

Figure 4. Alteration of attraction behavioral responses of wild type Canton-S flies fed with control diet and HFD for seven days and 14 days to several odors in a T-maze. Flies fed with HFD showed different responses in terms of attraction and repulsion to odorants compared with those fed with the control diet. 20 male flies were tested in each trial. The numbers of flies trapped in the control trap were indicated on the right side of the T-maze, while those in the test trap were indicated on the left side. ${ }^{*} p<0.05$.

\subsection{Alteration of Gene Expression Profiles by HFD Treatment}

While considering the modification in patterns of olfactory sensitivity and behavior to odorants, we subsequently examined the gene expression levels in the antenna from flies fed with HFD for 14 days. Our Illumina paired-end sequencing yielded a total of 13,287,258 and 11,103,566 raw reads with the length of $101 \mathrm{bp}$ from antenna tissue in flies fed with control diet (CD) and high fat diet (HFD), respectively. Firstly, we quantified our sequencing data by mapping against files transcript sequences. This analysis revealed that $72 \%$ of reads were mapped in the control diet-treated flies, while $67 \%$ were aligned in HFD-treated flies. Collectively, over two-thirds of the Drosophila melanogaster transcriptome data was expressed in both the control and HFD-treated flies, which is a similar observation for gene expression in other insects [26].

Then, we attempted to identify differentially expressed genes between control diet and HFD. A significant change in the level of gene expression after HFD treatment was indicated for a total of 732 genes, of which 379 genes were up-regulated and 343 genes down-regulated (Figure 5). From functional annotation, we noticeably identified genes that are related to olfactory related genes and neuronal sensor, which were down-regulated (Table 1). A total of 74 olfactory genes, 
including 34 odorant receptors (ORs) and 30 odorant-binding proteins (OBPs) were up-regulated and down-regulated from antennae of flies on high fat diet (Tables 2 and 3 ).

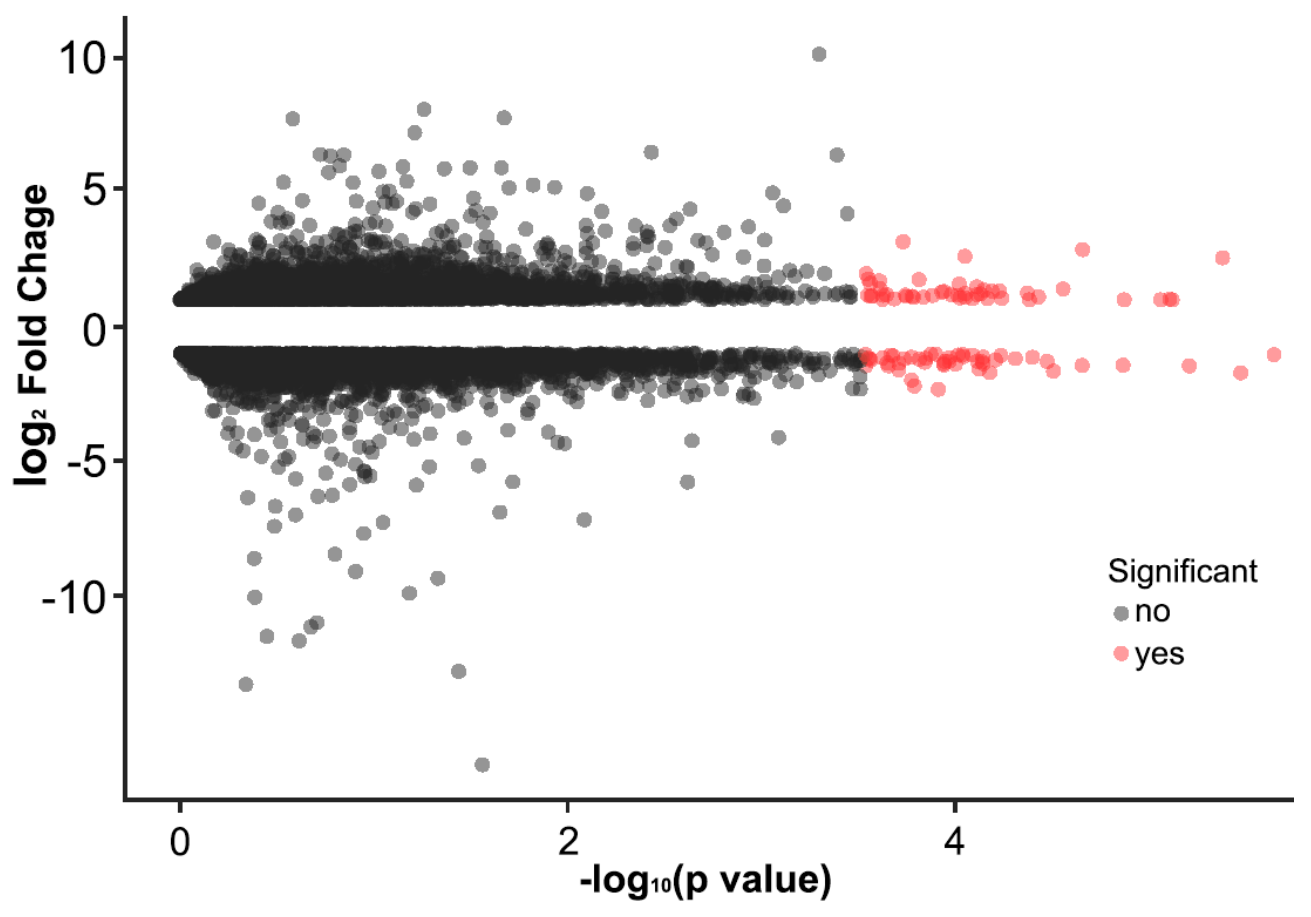

Figure 5. Gene expression changes in antennae of files on high fat diet. The $\mathrm{x}$-axis is the $-\log 10 p$-value and the $y$ axis is the fold-change value. Using the $p$-value 0.05 as the threshold cutoff, 97 genes in the left and right are selected. Red spots indicate the statistically significant differentially expressed genes (DEGs).

Table 1. Functional description for differentially expressed genes.

\begin{tabular}{cccc}
\hline Gene ID & Functional Description & Gene ID & Functional Description \\
\hline Upregulated & & Downregulated & \\
\hline FBtr0086664 & IM2 & FBtr0025595 & Akhr \\
FBtr0028381 & Decay & FBgn0052523 & Serine protease \\
FBtr0070099 & Cyp4g1 & FBgn0026389 & Or43a \\
FBtr0100231 & RpL41 & FBtr0082607 & GstD1 \\
FBtr0086662 & IM1 & FBtr0073062 & Drs15 \\
FBtr0074176 & sun & FBgn0053532 & Lectin-37Da \\
FBtr0088035 & eEF1 $\alpha 1$ & FBgn0037324 & Orco \\
FBtr0073468 & anthh & FBtr0085805 & RpL6 \\
FBtr0087992 & Cyp6g1 & FBgn0036926 & CG7646 \\
FBgn0001179 & Hay & FBgn0026385 & Or47b \\
FBtr0072924 & RpL8 & FBtr0074969 & lush \\
FBtr0307212 & CG16978 & FBtr0079455 & Obp28a \\
FBtr0086477 & Obp56d & FBtr0076229 & Sod1 \\
FBtr0072185 & RpL39 & FBgn0036009 & Or67a \\
FBgn0013343 & Syntaxin-1A & FBtr0081427 & CG9336 \\
FBgn0035505 & Teh2 & FBtr0081855 & COX7A \\
FBtr0113742 & RpL15 & FBtr0086292 & Obp57c \\
FBtr0086216 & CG18067 & FBtr0087494 & CG30197 \\
FBtr0081597 & Obp84a & FBgn0038798 & Or92a \\
FBgn0033483 & Egr & FBgn0034474 & Obp56g \\
FBtr0080306 & CG6770 & FBtr0071135 & RpS6 \\
FBtr0265464 & Traf6 & FBtr0078769 & RpL35A \\
\hline
\end{tabular}


Table 1. Cont.

\begin{tabular}{cccc}
\hline Gene ID & Functional Description & Gene ID & Functional Description \\
\hline Upregulated & & Downregulated & \\
\hline FBtr0084410 & RpS3 & FBgn0034475 & Obp56h \\
FBtr0300322 & Sfp84E & FBgn0033789 & CG13324 \\
FBtr0085463 & Obp99c & FBgn0038203 & Or88a \\
FBgn0053502 & CG33502 & FBtr0088525 & RpL31 \\
FBtr0070983 & RpL17 & FBtr0082370 & RpS25 \\
FBtr0087747 & CG4716 & FBtr0086672 & GstE4 \\
FBgn0041625 & Or65a & FBgn0028416 & Met75Ca \\
FBgn0036078 & Or67c & FBtr0071935 & RpS24 \\
FBtr0071096 & RpS14b & FBtr0075217 & CG7630 \\
FBtr0075884 & RpS4 & FBgn0032008 & CG14277 \\
FBtr0300635 & CG42502 & FBtr0070290 & sta \\
FBtr0087105 & RpLP2 & FBtr0307166 & Obp19a \\
FBtr0072805 & RpL23A & FBtr0302899 & CG6503 \\
FBtr0305965 & CG14661 & FBtr0073097 & RpL28 \\
FBtr0075839 & Nplp2 & FBtr0078656 & Obp83a \\
FBtr0075955 & Obp69a & FBtr0072164 & EbpIII \\
FBtr0075066 & RpL26 & FBtr0112740 & ND-MWFE \\
FBtr0077470 & RpL40 & FBtr0071392 & CG9691 \\
FBtr0076423 & RpS9 & FBtr0307366 & lncRNA:CR34335 \\
FBtr0082962 & His4r & FBtr040734 & CG15065 \\
FBtr0081920 & CG8369 & FBtr0077922 & a5 \\
FBtr0072173 & eEF5 & FBtr0040735 & CG16386 \\
FBtr0071360 & RpS28b & FBtr0083969 & RpS30 \\
FBtr0076273 & CG6409 & FBtr0075290 & a10 \\
FBtr0078655 & Obp83b & FBtr0300828 & RpS15Aa \\
FBtr0300321 & Os-C & FBtr0076032 & RpL10Ab \\
& & FBtr0332183 & OS9 \\
\hline & & &
\end{tabular}

Table 2. Olfactory receptor (Or) genes: Down- and up-regulated after 14 days HFD treatment.

\begin{tabular}{|c|c|c|c|}
\hline Gene ID & Functional Description & Gene ID & Functional Description \\
\hline Upregulated & & Downregulated & \\
\hline FBgn0026398 & Or22a & FBgn0037324 & Orco \\
\hline FBgn0026397 & Or22b & FBgn0030204 & Or9a \\
\hline FBgn0041625 & Or65a & FBgn0041626 & Or19a \\
\hline FBgn0036078 & Or67c & FBgn0062565 & Or19b \\
\hline FBgn0037399 & Or83c & FBgn0026395 & Or23a \\
\hline \multirow[t]{22}{*}{ FBgn0037685 } & Or85f & FBgn0026392 & Or33a \\
\hline & & FBgn0026391 & Or33b \\
\hline & & FBgn0028946 & Or35a \\
\hline & & FBgn0033041 & Or42a \\
\hline & & FBgn0033043 & Or $42 b$ \\
\hline & & FBgn0026389 & Or43a \\
\hline & & FBgn0033404 & Or45a \\
\hline & & FBgn0026386 & Or47a \\
\hline & & FBgn0026385 & Or47b \\
\hline & & FBgn0028963 & Or49b \\
\hline & & FBgn0034473 & Or56a \\
\hline & & FBgn0041624 & Or65b \\
\hline & & FBgn0041623 & Or65c \\
\hline & & FBgn0036009 & Or67a \\
\hline & & FBgn0036019 & Or67b \\
\hline & & FBgn0041622 & Or69a \\
\hline & & FBgn0037576 & Or85a \\
\hline & & FBgn0026399 & Or85e \\
\hline & & FBgn0038203 & Or88a \\
\hline & & FBgn0038798 & Or92a \\
\hline & & FBgn0039551 & Or98a \\
\hline & & FBgn0038798 & Or92a \\
\hline
\end{tabular}


Table 3. Odorant-binding protein (Obp) genes: Down- and up-regulated after 14 days HFD treatment.

\begin{tabular}{cccc}
\hline Gene ID & Functional Description & Gene ID & Functional description \\
\hline Upregulated & & Downregulated & \\
\hline FBgn0050067 & Obp50a & FBgn0030103 & Obp8a \\
FBgn0033931 & Obp50e & FBgn0030985 & Obp18a \\
FBgn0034468 & Obp56a & FBgn0031109 & Obp19a \\
FBgn0034470 & Obp56d & FBgn0031110 & Obp19b \\
FBtr0075955 & Obp69a & FBgn0031111 & Obp19c \\
FBgn0046876 & Obp83ef & FBgn0050052 & Obp49a \\
FBtr0078655 & Obp83b & FBgn0043530 & Obp51a \\
FBtr0081597 & Obp84a & FBgn0046879 & Obp56c \\
FBgn0039682 & Obp99c & FBgn0034471 & Obp56e \\
& & FBgn0043533 & Obp56f \\
& & FBgn0034474 & Obp56g \\
& & FBgn0034475 & Obp56h \\
& & FBgn0034509 & Obp57c \\
& & FBgn0050145 & Obp57e \\
& & FBgn0034768 & Obp58b \\
& & FBgn0034769 & Obp58c \\
& & FBgn0034770 & Obp58d \\
& & FBgn0034766 & Obp59a \\
& & FBgn0046875 & Obp83a \\
& & FBgn0039685 & Obp99b \\
& & FBgn0039684 & Obp99d \\
\hline & &
\end{tabular}

Next, we analyzed the expression of the genes associated with insulin signaling pathways (Table S1). Normalization of qRT-PCR was performed for four reference genes that are involved with insulin signaling genes to increase accuracy. We measured the transcript variation of DILP2, DILP3, DILP5, and InR genes for data normalization. Although the relative levels of DILP2, DILP3, and DILP5 expression showed no significant differences, InR expression demonstrated a significant decrease (Figure 6). 

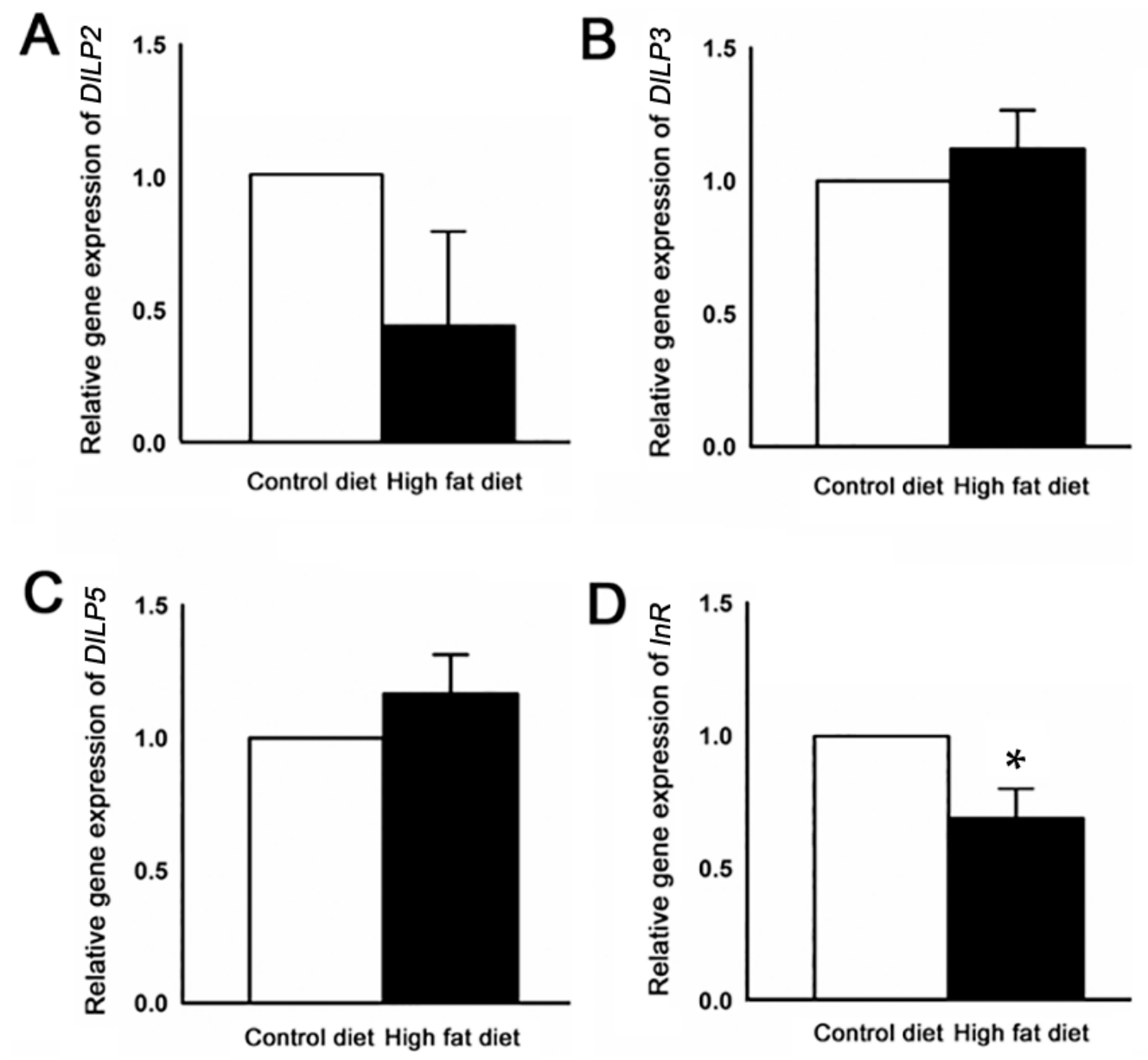

Figure 6. Gene expression levels of some target genes related to insulin signaling identified by DEG analysis. (A) Relative DILP2 mRNA transcript levels after different food treatments for 14 days. (B) Relative DILP3 and (C) DILP5 mRNA transcript levels after different food treatments for 14 days. Both of the gene expressions were increased by approximately $10 \%$ in high fat diet (HFD)-fed flies as compared with control flies. (D) Relative InR mRNA transcript levels after different food treatments for 14 days. InR gene expression level was significantly decreased by approximately $30 \%$ compared with control flies. All qRT-PCRs were carried out in triplicate $\left.{ }^{*} p<0.05\right)$. Error bars represent SEM.

To find out the main functional process that is affected by HFD treatment, we categorized the genes whose expression exhibited the substantial changes after HFD treatment. We analyzed the genes with a more than log two-fold change in expression in up- and down-regulation. These data were established according to the functional groups of GO, including biological process (BP), molecular function (MF), and cellular component (CC) (Figure 7). In particular, the largest number of genes was representative of several categories of BP, namely neurological system process, cognition, and sensory perception (Figure 7), which suggests a relevance to olfactory responses in both the peripheral and central nervous systems. Furthermore, genes of defense response and immune response were associated with response to HFD treatment. 


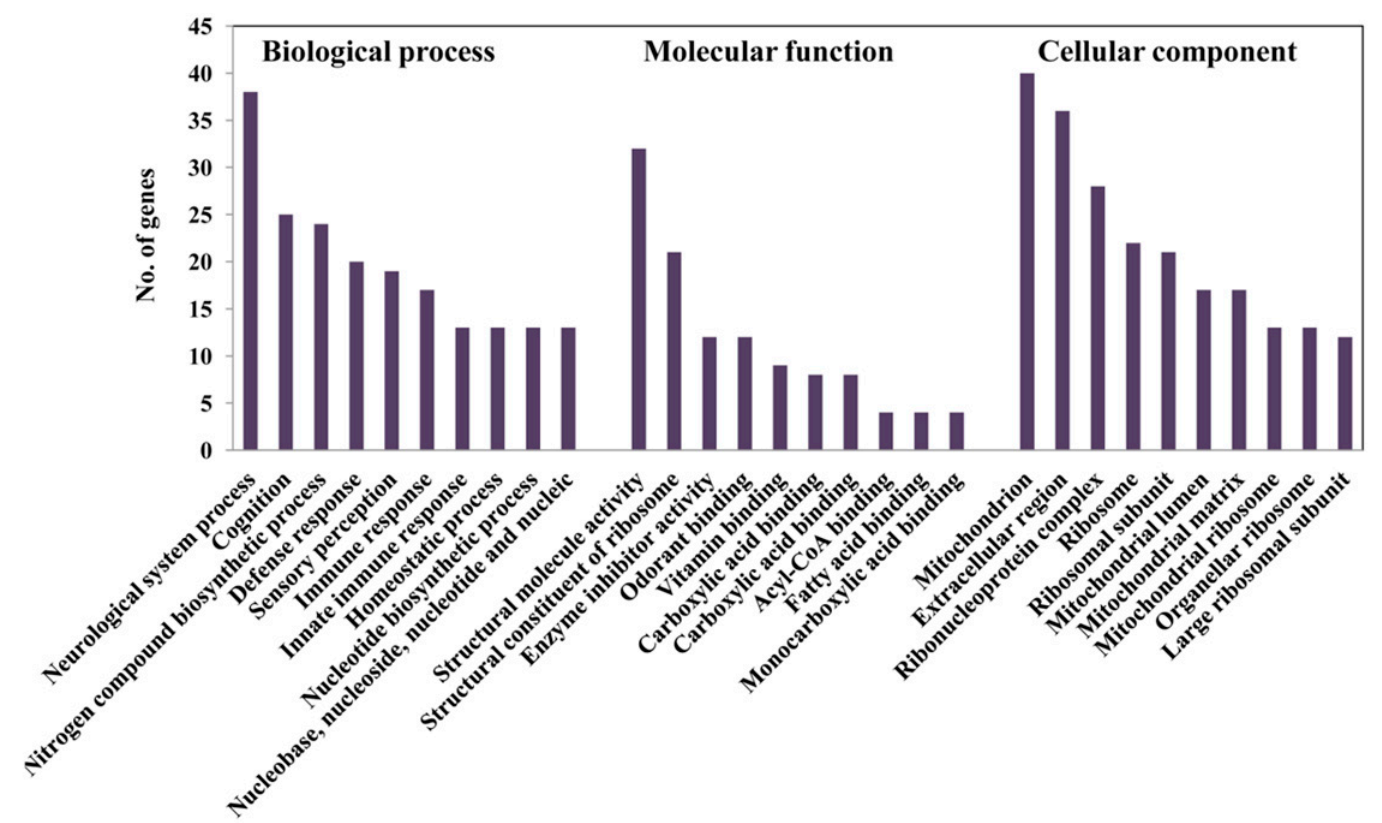

Figure 7. Gene Ontology (GO) analysis of expressed genes showing $\log _{2}$ fold changes between control diet (CD) and high fat diet (HFD). GO terms in the biological process, molecular function, and cellular component were shown.

\section{Discussion}

\section{A High-Fat Diet Leads to Olfactory Dysfunction in Homeostatic Processing in Drosophila}

High fat diet (HFD) is associated with an increased risk for disease, including obesity, heart disease, diabetes, and cancer [5-7]. In the present study, HFD for 14 days lead to a loss of climbing ability and reduction of life span of flies. Although consumption of HFD are found to cause nervous system dysfunction, whether such diets affect peripheral sensory system is largely unknown. We discovered a relationship between HFD and olfactory dysfunction in the fruit fly. Excessive stress by a HFD has a crucial impact on olfactory sensitivity. HFD-treated flies for 14 days were less sensitive to five food odors and three non-food odors. This is consistent with a recent reported that mice on HFD exhibited decreased olfactory discrimination [27]. This reduction in sensory sensitivity, which is exposed by a chronic fat diet, may be linked to the significantly decrease of olfactory related genes or other neuromodulators. For example, our DEG analysis indicated that 27 olfactory receptor genes and 21 odorant binding protein genes were down regulated in flies on fat diet. Because ORs and OBPs have been demonstrated to play an important role in olfactory receptor neurons maturation and axon guidance [28], one speculation is that high fat diet induced the down regulation of OR and OBP expression in olfactory receptor neuron that might thereby interfere with appropriate gene processing and targeting olfactory receptor neuronal axons to the proper antennal lobe region [29]. The cellular mechanisms underlying the HFD induced regulation of olfactory receptor gene expression are largely unknown. Recent evidence has emerged that proinflammatory responses are evoked in the olfactory epithelium in response to altered energy consumption [30]. HFD induce an increase in the number of macrophages and neuronal death, which resulted in a loss of connections from the olfactory epithelium and olfactory bulb [31]. Our DEG data showed that there was an upregulation of two genes that are known to be involved in apoptotic processes: Traf6 and Decay. Increased expression of these genes may induce apoptotic death in response to HFD. Further experiments are necessary to demonstrate whether Traf6 and Decay can induce apoptotic death of olfactory receptor neurons. Our DEG analysis provides the possibility that some mediators may regulate expression of the olfactory receptor genes and odorant binding proteins. Certain genes were observed in functional changes, which is associated with olfactory and nutrient-related pathways that modulate olfactory responses in flies. Previous 
studies demonstrated microarray analysis after starvation [32] or heat treatment [26] that affected olfactory responses and feeding behaviors, depending on nutritional status in adult flies. In both cases, the analysis included the antennal part, which is the main olfactory receptor organ, to make clear the importance of the internal state of olfaction.

One of the most prominent changes we found was a reduced appetitive behavioral response to olfactory stimuli, suggesting that HFD feeding may cause the alteration in olfactory perception. This is consistent with a recent report that mice with HFD-induced obesity showed deficits in olfactory learning and memory formation and altered behaviors related to olfaction and taste [33]. Besides, obese people have been shown to prefer a food-related odorant rather than a non-food related odorant when compared with normal weight people as same ages, implying that these people might not be able to regulate food intake by their internal state through appropriate odor processing [8]. Although molecular and neural mechanisms underlying the HFD-induced modification of olfactory behavior are not addressed yet, many lines of the research have been focused on insulin resistance. It has been reported that the activation of food stress responses is involved in the induction of insulin resistance and interestingly these results are observed in the olfactory bulb [34], implying that food stress-induced insulin resistance may affect the sensory cognition ability in animals.

In Drosophila, local signals by the neuropeptides and global metabolic cues by insulin are modified at specific olfactory sensory neuron to change olfactory responses [21,35,36]. For example, insulin as a metabolic factor modulates olfactory responses in fly antennae by enhancing the pre-synaptic facilitation and regulating odor preference in accordance with the internal state [18]. Furthermore, Drosophila insulin-like peptide 2 (DILP2) was shown to modulate a short neuropeptide F receptor (sNPFR1), which regulates olfactory driven behaviors in antenna and is also stimulated in starved flies [37-39]. Similarly, several studies demonstrate the importance of neuropeptides in the regulation of olfactory behavior $[40,41]$. Our digital gene expression analysis detected transcripts of DILP2 and insulin receptor $(I n R)$ in significant changes of down-regulation, in contrast with other insulin-related genes. This could be partially explained by the reduction of olfactory sensitivity caused by HFD treatment, and this may cause a loss of controlling ability of attractive behaviors to food odors. Recent research has demonstrated that DILP2 shows different expression levels depending on the type of diet [42]. Therefore, high fat diet condition must be function with other peripheral receptors, including those for olfaction. Olfactory responses to HFD treatment are affected by the over-expression of certain insulin signaling genes; therefore, communication through olfactory modulation could contribute to peripheral organs and subsequently be linked to the central nervous system $[13,43]$.

Interestingly, the behavioral responses of HFD-fed flies to benzaldehyde and pentyl acetate showed a transition between repulsive and attractive behavior, which is contrary to the expected physiological responses of the fly. This defective olfactory behavior might be associated with the misexpression of specific genes. Our DEG analysis showed that transcript levels of several olfactory receptor genes and odorant binding protein genes were changed. Among these genes, misexpression of the or $43 a$, one of the benzaldehyde receptors, caused a reduction of behavioral avoidance responses to benzaldehyde [44]. In addition, lush is a soluble odorant binding protein and it is expressed exclusively in the chemosensory system in flies. Mutants of this gene have been reported to a loss of the avoidance behaviors [45] and defects for pheromone-evoked behaviors [46]. Since the genetic dissection by which genes modulate olfactory behavior about each odorant remains unclear, further genetic experiments are necessary to demonstrate the relevance of specific olfactory sensory input and subsequent processing for olfactory driven behaviors.

\section{Materials and Methods}

\subsection{Drosophila Stocks and Diet Treatment}

The Canton-S wild-type strain of Drosophila melanogaster that was obtained from Bloomington Stock Center (Bloomington, IN, USA) was used in this study. All of the adult flies were maintained at 
$25{ }^{\circ} \mathrm{C}$ with $60 \%$ relative humidity and a $12 \mathrm{~h} \mathrm{light} / 12 \mathrm{~h}$ dark cycle. Food media for a standard diet was prepared by mixing $10 \%$ sugar, $10 \%$ yeast extract, and $1.5 \%$ agar with $1 \%$ Tween $80(w / v)$ added in case of toxicity. To make the high-fat diet, $2 \%$ palmitic acid $(w / v)$ and $1 \%$ Tween $80(w / v)$ were added into the standard diet in accordance with a previous study [22].

In order to obtain adult flies for the experiments, newly enclosed flies were collected into new bottles and kept for two days on the standard diet. Next day, male and female flies were sorted under $\mathrm{CO}_{2}$ anesthesia and only male flies were placed on the standard diet for one more day. Test male flies were treated with the standard diet and a high-fat diet (HFD) for seven days and 14 days, respectively. Each vial contained 20 male flies.

\subsection{Life Span and Climbing Assays}

For life span assays, flies were kept at a density of 20 male flies per each vial on the standard diet and HFD. Each group of flies was transferred to a vial with fresh food medium every three days and dead flies in each group were counted every day. Five replicates were conducted for this experiment.

For climbing assays, a behavioral paradigm that was reported in a previous study was employed with slight modifications [24]. Twenty male flies were transferred to a $10 \mathrm{~cm}$ glass vial and placed at the bottom of the vial by gently tapping the flies down to the bottom. The number of flies that climbed above $80 \%$ height within $30 \mathrm{~s}$ was counted. Experimental trials were repeated ten times independently for each group. The climbing index (\%) was calculated as the ratio of the number of flies climbing above $80 \%$ height to the total number of flies multiplied by 100 . All of the climbing assay experiments were performed at $25{ }^{\circ} \mathrm{C}$ with $60 \%$ humidity.

\subsection{Odor Stimulation}

All the odorants tested in this study (ethyl acetate, isoamyl acetate, pentyl acetate, benzaldehyde, 2-heptanone, 1-hexanol, 3-octanol, 1-octanol, 4-propyl phenol, and 4-methyl phenol) were commercially available products at the highest purity ( $>98 \%$, Sigma-Aldrich, Milwaukee, WI, USA). These odorants were dissolved in mineral oil (Sigma-Aldrich-330760) or ethanol at $1 \%$ dilution $(v / v)$. A glass tube was prepared with a continuous, humidified air stream, and $20 \mu \mathrm{L}$ of odorant solution was soaked onto a filter paper ( $4 \times 4 \mathrm{~mm}$, Toyo Roshi Kaisha, Tokyo, Japan) that was placed into a $5 \mathrm{~mL}$ disposable syringe with air pressure. These chemicals were used as odorant sources for EAG recordings and for behavioral assays.

\subsection{Electrophysiological Recordings}

Transepithelial electrophysiological recordings (electroantennograms, EAGs) from antennae in male Canton-S flies were made after individual diet treatment while using AgCl-coated silver wire inserted in a glass micropipette filled with $0.1 \mathrm{M} \mathrm{KCl}$. The experimental fly was immobilized in a truncated $200 \mu \mathrm{L}$ plastic pipette tip and trimmed to show an anterior aspect of the fly's head. The third segments of the antennae were exposed for EAG recordings. A reference electrode was inserted into a compound eye and a recording electrode was placed on the dorso-medial surface of the third antennal segment, as described previously [47]. Electrical signals were amplified with an analog $10 \times$ active probe and conveyed to an acquisition system (IDAC4, Syntech, Hilversum, The Netherlands). Signals were then further recorded and analyzed while using EAG Pro software (Syntech). A constant air stream of $40 \mathrm{~mL} / \mathrm{min}$ was delivered to the fly head by using a stimulus controller (CS-55, Syntech). Pulses of the odorants were produced during a $1 \mathrm{~s}$ stimulation through the Pasteur pipette to the syringe pipette. Signal amplitude of olfactory responses $(\mathrm{mV})$ was measured from a baseline before stimulation to the trough of the electrical signals after odorant stimulation. Control EAG experiments loaded with no odorant and mineral oil were used. The interval between each odorant stimulus was about $60 \mathrm{~s}$ to prevent the adaptation of the test animal to a given odorant. 


\subsection{Quantitative RT-PCR}

Flies were collected according to the procedures that are presented above for the diet treatment. Total RNA extraction was carried out while using an RNeasy Mini Kit (Qiagen, Valencia, CA, USA), according to the manufacturer's protocol. For each treatment, at least three independent extractions were conducted while using 100 fly antennae. The quality and quantity of total RNA was measured by a Nanodrop 2000 (Thermo Scientific Inc., Wilmington, DE, USA). cDNA was prepared from the extracted RNA while using the Superscript III First-Strand Synthesis kit (Invitrogen Inc., Carlsbad, CA, USA). Quantitative RT-PCR was performed using a StepOnePlus machine (Applied Biosystems Inc., Foster City, CA, USA) according to the manufacturer's protocol with SYBR green qRT-PCR Master Mix (Fermentas, Burlington, ON, Canada) to measure DmOrco gene expression. qRT-PCR measurement for each gene was repeated with three independent biological samples and quantitative analysis was conducted by StepOne plus Software V. 2.0 (Applied Biosystems). The transcript level of the gene was calculated by the standard curve method and normalized to the control gene ribosomal protein 49 (rp49) primer that was described in a previous paper [48]. The primer sequences for DmOrco were forward: 5'-GGTGGACCATGAGACGAACT-3'; reverse: 5'-CATCACGTCGCATAGATTGG-3'.

\subsection{Behavioral Assay}

Flies were placed under light $\mathrm{CO}_{2}$ anesthesia. Separated male flies were placed into vials (20 flies per vial) containing each diet treatment. Flies were transferred to new vials every two to three days and behavioral assays performed on days 7 and 14 after diet treatment. One day before the assay, flies were moved into empty vials containing only water on Kimwipes (Kimberly-Clark Worldwide, Neenah, WI, USA) for at least $15 \mathrm{~h}$ to produce a starved state [49]. For each measurement, the flies were gently tapped to place into a T-maze. The same odorants as used for the EAGs were used for this behavioral assay. Each vial at both sides contained two traps, an odor trap, and a control trap (water). Flies were allowed to have $30 \mathrm{~s}$ to choose one of the sides in the T-maze that contained odorant. The attraction index (AI) was calculated, as follows: (number of flies moving toward the odor trap number of flies in the control trap)/(total number of flies). Ten replicates were performed for each treatment. All T-maze assay experiments were conducted at $25^{\circ} \mathrm{C}$ with $60 \%$ humidity.

\subsection{Analysis for Differentially Expressed Genes (DEG) and Gene Ontology of HFD-Fed Flies}

Approximately 3000 samples of third antennal segments of male adult flies were prepared to achieve a sufficient representation of genes. The flies in each diet treatment were collected from 11:00 to 14:00 and 16:00 to 19:00 to prevent the possible modification of gene expression by circadian rhythms [26]. All the samples were incorporated to minimize any random factors that affect gene expression other than the treatment. Gene sequences were downloaded from the Drosophila Genome database (www.flybase.org) for the analysis of differentially expressed genes (DEG).

For the RNA sequencing experiment, $4 \mu \mathrm{g}$ of total RNA was extracted while using the same protocol, as described above in the quantitative RT-PCR procedure. In order to convert mRNA in total RNA into a library of template molecules for subsequent cluster generation, a Illumina ${ }^{\circledR}$ TruSeq $^{\mathrm{TM}}$ RNA sample preparation kit was used in accordance with the manufacturer's instructions (Illumina Inc., San Diego, CA, USA). The total RNA sample was used for poly-A mRNA selection using poly-T oligo-attached magnetic beads with two rounds of purification. The resulting mRNA sample from the antennae was subjected to thermal mRNA fragmentation while using Elute, Prime, Fragment Mix from the Illumina ${ }^{\circledR}$ TruSeq ${ }^{\mathrm{TM}}$ RNA sample preparation kit. The mRNA fragments were reverse transcribed to synthesize the first-strand cDNA using a combination of reverse transcriptase and random primers. The mRNA template strand was removed and double-stranded cDNA (ds cDNA) was generated using DNA polymerase I. The ds cDNA was purified using Ampure XP beads to separate the ds cDNA from the second-strand reaction mix. 
The cDNA fragments were then blunt-ended through an end-repair reaction while using an End Repair (ERP) mix (Illumina Inc). The $3^{\prime}$ to $5^{\prime}$ exonuclease activity of this mix removed the $3^{\prime}$ overhangs

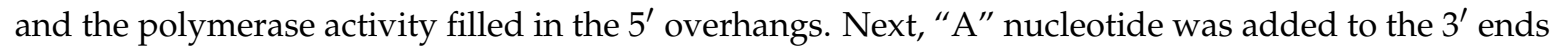
of the blunt fragments to prevent them from ligating to each other and then ligated to platform-specific double-stranded bar-coded adapters that provided a complementary overhang during the adapter ligation reaction. DNA libraries were sequenced using an Illumina HiSeq2000. The sequencing reads were deposited to the NCBI SRA (Short Read Archive) site (https: / / www.ncbi.nlm.nih.gov/sra) under the accession number SRR1501071 for the control diet and SRR1501074 for the high fat diet.

DEG analysis was performed using edgeR bioconductor package and RSEM software [50]. Quality check, normalization, and statistical analysis were performed to develop significantly matched signals. Genes with false discovery rate (FDR) value of at most 0.001 and fold change value of $\geq 2$ were considered as significant differentially expressed genes. In addition, $\log _{2}$ (FPKM) expressional difference was manually calculated for all of the genes based on FPKM (Fragments Per Kilobase Million) values. After obtaining general information on the gene expression, gene ontology (GO) analysis was carried out for genes that showed $\log _{2}$ fold digital gene expression difference after diet treatment using the DAVID tool (http:/ / david.abcc.ncifcrf.gov/). In the DAVID annotation system, modified Fisher Exact $p$ value (EASE score) was adopted to measure the gene-enrichment in annotation terms. All data analysis and visualization of differentially expressed genes were conducted while using R 2.15.1 (http:/ / www.r-project.org).

\subsection{Statistical Analysis}

Statistical analyses were performed by Student's t-test (SPSS, Version 20, IBM, New York, NY, USA) on the results from the lifespan assay, climbing ability test, EAGs, and T-maze tests to determine any significant differences between the control diet and HFD treatment. Data were shown as means \pm standard error.

\section{Conclusions}

High-fat diet affects sensory abilities in Drosophila melanogaster such as olfactory-driven behaviors and olfactory sensitivity to several odorants, which in turn makes some changes in molecular levels of gene expressions. Integration of internal and external factors provides crucial evidence to olfactory modulation in animals. Our present study demonstrates that high fat diet alters olfactory perception and changes of gene expression profiles in Drosophila melanogaster. A better understanding of the complex mechanisms that are underlying olfactory modulation might help to characterize the olfactory systems that are affected by HFD treatment. Identifying novel regulators of the olfactory system and examining them to find out specific odorant receptors to odors at the intracellular level affected by HFD treatment may provide an understanding of the mechanisms and circuits from antenna to brain in flies.

Supplementary Materials: Supplementary materials can be found at http://www.mdpi.com/1422-0067/19/10/ 2855/s1.

Author Contributions: J.J., D.-I.K., G.-Y.H., and H.W.K. designed experiments and J.J., D.-I.K., and G.-Y.H. carried out the experiments. J.J. and H.W.K. prepared the manuscript.

Funding: This research was supported by Basic Science Research Program through the National Research Foundation of Korea (NRF) funded by the Ministry of Science, ICT \& Future Planning (2016R1A2B3011742) and Cooperative Research Program for Rural Development Administration (Project No. PJ012526012018). This study also partly supported by Incheon National University internal grant (2016) to H.K.

Acknowledgments: We are grateful to Murukarthick Jayakodi assisting a differentially expressed gene (DEG) analysis and to Soo Ho Lim for helping graphical art work.

Conflicts of Interest: The authors declare no conflict of interest. 


\section{References}

1. Rajan, A.; Perrimon, N. Drosophila as a model for interorgan communication: Lessons from studies on energy homeostasis. Dev. Cell 2011, 21, 29-31. [CrossRef] [PubMed]

2. Rajan, A.; Perrimon, N. Of flies and men: Insights on organismal metabolism from fruit flies. BMC Biol. 2013, 11, 38. [CrossRef] [PubMed]

3. Baker, K.D.; Thummel, C.S. Diabetic larvae and obese flies-emerging studies of metabolism in Drosophila. Cell Metab. 2007, 6, 257-266. [CrossRef] [PubMed]

4. Skorupa, D.A.; Dervisefendic, A.; Zwiener, J.; Pletcher, S.D. Dietary composition specifies consumption, obesity, and lifespan in Drosophila melanogaster. Aging Cell 2008, 7, 478-490. [CrossRef] [PubMed]

5. Gale, S.M.; Castracane, V.D.; Mantzoros, C.S. Energy homeostasis, obesity and eating disorders: Recent advances in endocrinology. J. Nutr. 2004, 134, 295-298. [CrossRef] [PubMed]

6. Trinh, I.; Boulianne, G.L. Modeling obesity and its associated disorders in Drosophila. Physiology 2013, 28, 117-124. [CrossRef] [PubMed]

7. Teleman, A.A.; Ratzenbock, I.; Oldham, S. Drosophila: A model for understanding obesity and diabetic complications. Exp. Clin. Endocrinol. Diabetes 2012, 120, 184-185. [CrossRef] [PubMed]

8. Thompson, D.A.; Moskowitz, H.R.; Campbell, R.G. Taste and olfaction in human obesity. Physiol. Behav. 1977, 19, 335-337. [CrossRef]

9. Withcher, D. Metabolic regulation and behavior: How hunger produces arousal-an insect study. Endocr. Metab. Immune 2007, 7, 304-310.

10. Crowley, V.E. Overview of human obesity and central mechanisms regulating energy homeostasis. Ann. Clin. Biochem. 2008, 45, 245-255. [CrossRef] [PubMed]

11. Melcher, C.; Bader, R.; Pankratz, M.J. Amino acids, taste circuits, and feeding behavior in Drosophila: Towards understanding the psychology of feeding in flies and man. J. Endocrinol. 2007, 192, 467-472. [CrossRef] [PubMed]

12. Herrero, P. Fruit fly behavior in response to chemosensory signals. Peptides 2012, 38, 228-237. [CrossRef] [PubMed]

13. Sato, K.; Touhara, K. Insect olfaction: Receptors, signal transduction, and behavior. Results Probl. Cell Differ. 2009, 47, 121-138. [PubMed]

14. Carlson, J.R. Olfaction in Drosophila: From odor to behavior. Trends Genet. 1996, 12, 175-180. [CrossRef]

15. Lacroix, M.C.; Badonnel, K.; Meunier, N.; Tan, F.; Schlegel-Le Poupon, C.; Durieux, D.; Monnerie, R.; Baly, C.; Congar, P.; Salesse, R.; et al. Expression of insulin system in the olfactory epithelium: First approaches to its role and regulation. J. Neuroendocrinol. 2008, 20, 1176-1190. [CrossRef] [PubMed]

16. Vosshall, L.B. Into the mind of a fly. Nature 2007, 450, 193-197. [CrossRef] [PubMed]

17. Diop, S.B.; Bodmer, R. Drosophila as a model to study the genetic mechanisms of obesity-associated heart dysfunction. J. Cell. Mol. Med. 2012, 16, 966-971. [CrossRef] [PubMed]

18. Root, C.M.; Ko, K.I.; Jafari, A.; Wang, J.W. Presynaptic facilitation by neuropeptide signaling mediates odor-driven food search. Cell 2011, 145, 133-144. [CrossRef] [PubMed]

19. Palouzier-Paulignan, B.; Lacroix, M.C.; Aime, P.; Baly, C.; Caillol, M.; Congar, P.; Julliard, A.K.; Tucker, K.; Fadool, D.A. Olfaction under metabolic influences. Chem. Senses 2012, 37, 769-797. [CrossRef] [PubMed]

20. Nassel, D.R.; Winther, A.M. Drosophila neuropeptides in regulation of physiology and behavior. Prog. Neurobiol. 2010, 92, 42-104. [CrossRef] [PubMed]

21. Libert, S.; Zwiener, J.; Chu, X.; Vanvoorhies, W.; Roman, G.; Pletcher, S.D. Regulation of Drosophila life span by olfaction and food-derived odors. Science 2007, 315, 1133-1137. [CrossRef] [PubMed]

22. Sun, X.; Seeberger, J.; Alberico, T.; Wang, C.; Wheeler, C.T.; Schauss, A.G.; Zou, S. Acai palm fruit (Euterpe oleracea Mart.) pulp improves survival of flies on a high fat diet. Exp. Gerontol. 2010, 45, 243-251. [CrossRef] [PubMed]

23. Boyd, O.; Weng, P.; Sun, X.; Alberico, T.; Laslo, M.; Obenland, D.M.; Kern, B.; Zou, S. Nectarine promotes longevity in Drosophila melanogaster. Free Radic. Biol. Med. 2011, 50, 1669-1678. [CrossRef] [PubMed]

24. Birse, R.T.; Choi, J.; Reardon, K.; Rodriguez, J.; Graham, S.; Diop, S.; Ocorr, K.; Bodmer, R.; Oldham, S. High-fat-diet-induced obesity and heart dysfunction are regulated by the TOR pathway in Drosophila. Cell Metab. 2010, 12, 533-544. [CrossRef] [PubMed] 
25. Leinwand, S.G.; Chalasani, S.H. Olfactory networks: From sensation to perception. Curr. Opin. Genet. Dev. 2011, 21, 806-811. [CrossRef] [PubMed]

26. Riveron, J.; Boto, T.; Alcorta, E. Transcriptional basis of the acclimation to high environmental temperature at the olfactory receptor organs of Drosophila melanogaster. BMC Genomics 2013, 14, 259. [CrossRef] [PubMed]

27. Takase, K.; Tsuneoka, Y.; Oda, S.; Kuroda, M.; Funato, H. High-fat diet feeding alters olfactory-, social-, and reward-related behaviors of mice independent of obesity. Obesity 2016, 24, 886-894. [CrossRef] [PubMed]

28. Feinstein, P.; Bozza, T.; Rodriguez, I.; Vassalli, A.; Mombaerts, P. Axon guidance of mouse olfactory sensory neurons by odorant receptor sand the $\beta 2$ adrenergic receptor. Cell 2004, 117, 833-846. [CrossRef] [PubMed]

29. Barish, S.; Volkan, P.C. Mechanisms of olfactory receptorneuron specification in Drosophila. WIREs. Dev. Biol. 2015, 4, 609-621. [CrossRef] [PubMed]

30. Gregor, M.F.; Hotamisligil, G. Inflammatory mechanisms in obesity. Ann. Rev. Immunol. 2011, $29,415-445$. [CrossRef] [PubMed]

31. Moraes, J.C.; Coope, A.; Morari, J.; Cintra, D.E.; Roman, E.A.; Pauli, J.R.; Romanatto, T.; Carvalheira, J.B.; Oliveira, A.L.R.; Saad, M.J.; et al. High-fat diet induces apoptosis of hypothalamic neurons. PLoS ONE 2009, 4, e5045. [CrossRef] [PubMed]

32. Farhadian, S.F.; Suarez-Farinas, M.; Cho, C.E.; Pellegrino, M.; Vosshall, L.B. Post-fasting olfactory, transcriptional, and feeding responses in Drosophila. Physiol. Behav. 2012, 105, 544-553. [CrossRef] [PubMed]

33. Thiebaud, N.; Johnson, M.C.; Butler, J.L.; Bell, G.A.; Ferguson, K.L.; Fadool, A.R.; Fadool, J.C.; Gale, A.M.; Gale, D.S.; Fadool, D.A. Hyperlipidemic diet causes loss of olfactory sensory neurons, reduces olfactory discrimination, and disrupts odor-reversal learning. J. Neurosci. 2014, 34, 6970-6984. [CrossRef] [PubMed]

34. Fadool, D.A.; Tucker, K.; Pedarzani, P. Mitral cells of the olfactory bulb perform metabolic sensing and are disrupted by obesity at the level of the Kv1.3 ion channel. PLoS ONE 2011, 6, e24921. [CrossRef] [PubMed]

35. Musselman, L.P.; Fink, J.L.; Narzinski, K.; Ramachandran, P.V.; Hathiramani, S.S.; Cagan, R.L.; Baranski, T.J. A high-sugar diet produces obesity and insulin resistance in wild-type Drosophila. Dis. Mod. Mech. 2011, 4, 842-849. [CrossRef] [PubMed]

36. Heinrichsen, E.T.; Haddad, G.G. Role of high-fat diet in stress response of Drosophila. PLoS ONE 2012, 7, e42587. [CrossRef] [PubMed]

37. Morris, S.N.; Coogan, C.; Chamseddin, K.; Fernandez-Kim, S.O.; Kolli, S.; Keller, J.N.; Bauer, J.H. Development of diet-induced insulin resistance in adult Drosophila melanogaster. Biochim. Biophys. Acta 2012, 1822, 1230-1237. [CrossRef] [PubMed]

38. Benton, R. On the ORigin of smell: Odorant receptors in insects. Cell. Mol. Life Sci. 2006, 63, 1579-1585. [CrossRef] [PubMed]

39. Lee, K.S.; Kwon, O.Y.; Lee, J.H.; Kwon, K.; Min, K.J.; Jung, S.A.; Kim, A.K.; You, K.H.; Tatar, M.; $\mathrm{Yu}, \mathrm{K}$. Drosophila short neuropeptide F signalling regulates growth by ERK-mediated insulin signalling. Nat. Cell Biol. 2008, 10, 468-475. [CrossRef] [PubMed]

40. Winther, A.M.; Acebes, A.; Ferrus, A. Tachykinin-related peptides modulate odor perception and locomotor activity in Drosophila. Mol. Cell. Neurosci. 2006, 31, 399-406. [CrossRef] [PubMed]

41. Kahsai, L.; Kapan, N.; Dircksen, H.; Winther, A.M.; Nassel, D.R. Metabolic stress responses in Drosophila are modulated by brain neurosecretory cells that produce multiple neuropeptides. PLoS ONE 2010, 5, e11480. [CrossRef] [PubMed]

42. Carlsson, M.A.; Diesner, M.; Schachtner, J.; Nassel, D.R. Multiple neuropeptides in the Drosophila antennal lobe suggest complex modulatory circuits. J. Comp. Neurol. 2010, 518, 3359-3380. [CrossRef] [PubMed]

43. Carlsson, M.A.; Enell, L.E.; Nassel, D.R. Distribution of short neuropeptide F and its receptor in neuronal circuits related to feeding in larval Drosophila. Cell Tissue Res. 2013, 353, 511-523. [CrossRef] [PubMed]

44. Störtkuhl, K.F.; Kettler, R.; Fischer, S.; Hovemann, B. An increased receptive field of olfactory receptor Or43a in the antennal lobe of Drosophila reduces benzaldehyde-driven avoidance behavior. Chem. Senses 2005, 30, 81-87. [CrossRef] [PubMed]

45. Kim, M.S.; Repp, A.; Smith, D.P. LUSH odorant-binding protein mediates chemosensory responses to alcohols in Drosophila melanogaster. Genetics 1998, 150, 711-721. [PubMed]

46. Xu, P.; Atkinson, R.; Jones, D.N.M.; Smith, D.P. Drosophila OBP LUSH is required for activity of pheromone-sensitive neurons. Neuron 2005, 45, 193-200. [CrossRef] [PubMed]

47. Tom, W.; de Bruyne, M.; Haehnel, M.; Carlson, J.R.; Ray, A. Disruption of olfactory receptor neuron patterning in Scutoid mutant Drosophila. Mol. Cell. Neurosci. 2011, 46, 252-261. [CrossRef] [PubMed] 
48. Hong, S.H.; Lee, K.S.; Kwak, S.J.; Kim, A.K.; Bai, H.; Jung, M.S.; Kwon, O.Y.; Song, W.J.; Tatar, M.; Yu, K. Minibrain/Dyrk1a regulates food intake through the Sir2-FOXO-sNPF/NPY pathway in Drosophila and mammals. PLoS Genet. 2012, 8, e1002857. [CrossRef]

49. Min, S.; Ai, M.; Shin, S.A.; Suh, G.S. Dedicated olfactory neurons mediating attraction behavior to ammonia and amines in Drosophila. Proc. Natl. Acad. Sci. USA 2013, 110, E1321-E1329. [CrossRef] [PubMed]

50. Trapnell, C.; Roberts, A.; Goff, L.; Pertea, G.; Kim, D.; Kelley, D.R.; Pimentel, H.; Salzberg, S.L.; Rinn, J.L.; Pachter, L. Differential gene and transcript expression analysis of RNA-seq experiments with TopHat and Cufflinks. Nat. Protoc. 2012, 7, 562-578. [CrossRef] [PubMed]

(C) 2018 by the authors. Licensee MDPI, Basel, Switzerland. This article is an open access article distributed under the terms and conditions of the Creative Commons Attribution (CC BY) license (http://creativecommons.org/licenses/by/4.0/). 